\title{
Enfermos pobres: Clasificaciones estatales, diferenciación social y acceso al bienestar en los años treinta (Córdoba-Argentina)
}

Artículo recibido: 14 de febrero de 2019

Aprobación final: 23 de agosto de 2019

\section{Resumen}

Las agencias estatales en contacto con la población ejecutan rutinariamente operaciones de clasificación destinadas a diferenciar a las personas y modelar su acceso a bienes materiales y simbólicos. Ahondando sobre esta acción burocrática, en este artículo ${ }^{1}$ analizamos las categorías estatales elaboradas para definir a los beneficiarios de las noveles políticas sanitarias construidas a partir de los años 1930, indagando quiénes fueron los encargados de crearlas, a quiénes incluyeron y excluyeron y qué sentidos les fueron otorgados. La exploración de esos procedimientos clasificatorios nos permitirá constatar que la utilización de viejas categorías sociales como las de "pobre" y "pudiente" sustentaron tempranos y duraderos procesos de focalización de las políticas de salud pública.

1 Versiones previas de este trabajo se beneficiaron de los comentarios realizados por diversos colegas y por los dictámenes de los evaluadores anónimos. En especial quiero recordar los comentarios realizados por Juan Suriano, quien en las VI Jornadas Nacional de Historia Social (2017) me alentó a continuar mis indagaciones sobre esta investigación. Sus agudas interpretaciones han sido extremadamente valiosas para quienes nos interesamos por la historia social argentina y su pérdida deja un vacío difícil de llenar. 


\title{
Sick Poor: State Classifications, Social Differentiation and Access to Welfare in the 1930s (Córdoba - Argentina)
}

\begin{abstract}
In contact with the population, the State agencies routinely perform classification operations aimed to differentiate people and to shape their access to material and symbolic goods. Going farther in the study of this operation, in this article we analyze the bureaucratic categories elaborated to define the beneficiaries of the novel health policies built since the 30s, investigating those who were responsible for creating them, whom they included and excluded and what senses were granted to them. The exploration of these procedures of classification allows to verify that the use of old social categories such as "pobre" and "pudientes" sustained early and durables processes of targeted health policies in Argentina.
\end{abstract}

Keywords: Social Classification, beneficiaries, Health Policies, Peronismo, Social Groups.

"Si es Ud. Pudiente y se asiste en el hospital, sepa que defrauda al pobre, al estado y al médico." ${ }^{2}$

"Se hace saber al público que los servicios del Dispensario -que son de carácter gratuito- se prestarán exclusivamente al sector menesteroso de la población; no debiendo concurrir aquellas personas cuya situación económica les permita solventar la atención médica que necesiten." ${ }^{3}$

“Clasificar no significa únicamente constituir grupos: significa disponer esos grupos de acuerdo a relaciones muy especiales. Nosotros los representamos como coordinados o subordinados los unos a los otros, decimos que éstos (las especies) están incluidos en aquéllos (los géneros), que los segundos subsumen a los primeros. Los hay que dominan, otros que son dominados, otros que son independientes los unos de los otros. Toda clasificación implica un orden jerárquico del que ni el mundo sensible ni nuestra conciencia nos brindan el modelo."

(Mauss y Durkheim, 1996: 30)

Las agencias estatales utilizan diferentes etiquetas para clasificar a la población en formularios, procedimientos y disposiciones que los burócratas cotidianamente imponen a la ciudadanía. Saturadas de jerarquías, privilegios, prescripciones, restricciones, habilitaciones y derechos heterogéneos, esas nociones disponen

2 "Recientemente el Colegio Médico de la Capital Federal, ha hecho circular en aquella ciudad con profusión, el affiche que reproducimos [...] Por creerlo de palpitante actualidad, así como de aplicación directa en los procedimientos de nuestra ciudad, no titubeamos en difundir dicha iniciativa, ya que ella entraña una profunda verdad y una lógica defensa de los intereses de la gente humilde." (26 de julio de 1933), "La asistencia hospitalaria", Los Principios, Córdoba, p. 2.

3 Comunicado de la dirección del Dispensario Cardiológico Nacional, dependiente de la Universidad Nacional de Córdoba. (6 de diciembre de 1946), "La atención en el dispensario de cardiología", Los Principios, Córdoba, p. 4. 
lugares por los que circular, comportamientos modélicos, tratamientos específicos y oportunidades particulares. Pero su eficacia surge de su doble naturaleza. No solo se objetivan en las estructuras estatales, sino también en las conciencias individuales. Las clasificaciones sociales suponen esquemas de percepción, apreciación y acción que se interiorizan y hacen de un determinado orden -con sus separaciones, agrupamientos y desigualdades- un esquema lógico y moral evidente, una doxa (Corrigan y Sayer, 2007 [1985]; Bourdieu, 1998; Lamont y Virág, 2002; entre otros).

En los últimos años, antropólogos e historiadores han realizado un importante trabajo develando las clasificaciones raciales, étnicas, sexuales, etarias, espaciales y políticas que estructuran las relaciones sociales y modelan la participación pública, la consideración social y el acceso a los recursos materiales y simbólicos en la Argentina contemporánea (Adamosvky, 2009; Grimson, 2017; Auyero, 2013, entre otros). Esta literatura se ha expandido bajo el supuesto de que el abordaje de la historicidad y la conflictividad subyacente a la construcción de estas categorías permite desnaturalizar las miradas sobre los grupos sociales y reconocer las relaciones de dominación cristalizadas en los aparatos estatales. Una agenda destinada a repensar las categorías sociales abordando críticamente sus externalidades, sus consecuencias e impactos en el pasado y en el futuro (De la Cadena, 2007: 8).

En línea con estos estudios, en este trabajo proponemos una historia de las políticas públicas que analice las clasificaciones que modelaron sus intervenciones, sus nociones de derecho, de beneficio y beneficiario, los límites de inclusión y exclusión que impusieron entre los grupos sociales y los mecanismos mediante los cuales delimitaron fronteras que definieron el usufructo de los bienes públicos. Esto implica observar a las agencias estatales argentinas como espacios en los que se construyeron mecanismos de diferenciación y discriminación. Para ello, nos interrogamos respecto a las clasificaciones estatales elaboradas para identificar a quienes debían ser los beneficiarios de las políticas de salud instituidas a partir de la entreguerras y los sentidos que les otorgaron diferentes actores históricos en esa etapa. Para llevar a cabo esa exploración realizamos un análisis en profundidad de un caso, el sistema de salud público de la provincia de Córdoba en los años treinta.

Nuestro supuesto principal es que las políticas sanitarias instituidas en esta provincia fueron conformadas a partir de la utilización de viejas clasificaciones sociales que impusieron restricciones sobre sus potenciales beneficiarios y aseguraron su temprana y duradera focalización. Esas prestaciones asistenciales se orientaron hacia una clientela construida sobre las nociones de "pobre", "necesitado", "de escasos recursos" y "humilde" y alrededor de las mismas fueron impuestos diversos cierres materiales y simbólicos que buscaron desalentar, cuando no prohibir, el acceso a quienes estuvieran en condiciones materiales para pagar la medicina privada, los enfermos "pudientes". En el transcurso del siglo XX, ese binomio constituyó una clasificación flexible cuyo contenido y los sujetos a los que identificó fueron variando de acuerdo a los contextos socioeconómicos, los ingresos 
de la población, los costos de la medicina y la generación de sistemas de servicios médicos privados que redefinieron las condiciones de acceso de la población a los servicios curativos -mutuales, obras sociales y empresas de servicios médicos-. De todos modos, subsistió una delimitación de la población modelo destinataria de la medicina curativa pública definida por sus carencias y arraigada en decretos, rutinas burocráticas y también en la cultura popular.

El análisis entonces del diseño e implementación de políticas sanitarias permite aseverar que la distribución de bienes públicos se construyó sobre la dicotomía "pobre"/"pudiente" y quienes necesitaron o desearon acceder a la oferta de salud estatal debieron asumir primero esa identidad social y adoptar sus prescripciones. Esto implicó que, para hacerse atender en los dispositivos públicos de salud, primero fue necesario ser investido de la condición de "pobre", "humilde”, "de escasos recursos" o "necesitado", asumir los atributos sociales correspondientes -hacerlos cuerpo mediante apariencias, actitudes y modos de tratamiento- e, incluso, en algunos casos, cumplir con procedimientos administrativos que certificaran esa particular naturaleza.

El análisis se centra en las políticas sanitarias construidas en Córdoba en la década de 1930 a través de un relevamiento de la producción legislativa y burocrática del estado provincial. Posteriormente intentamos explorar tentativamente algunos de los rasgos que adquirió este fenómeno durante la década peronista. Para el estudio fue realizado un relevamiento empírico exhaustivo de diferente tipo de documentos en los que se discutieron, criticaron, institucionalizaron y difundieron esas clasificaciones estatales. Esto remite a reglamentos, diarios de sesiones legislativos, documentación administrativa, planes públicos, memorias oficiales, leyes y decretos. También relevamos publicaciones oficiales de entidades médicas, la Revista Médica del Circulo Médico de Córdoba y la Revista del Colegio de Médicos, en donde los profesionales discutieron la demanda y oferta de prestaciones asistenciales, y la prensa periódica, un espacio en los que se debatieron nociones sobre quiénes podían y debían beneficiarse de los servicios de salud estatales (La Voz del Interior, Los Principios, Córdoba, La Nación, La Prensa, Crítica, El Mundo, El Día, El Diario, El Liberal, El Litoral, El Orden, El Progreso, El Radical, El Ambato, La Gaceta, Los Andes). Por último, relevamos un corpus heterogéneo de materiales como películas de época y publicaciones como Mundo Peronista, con la finalidad de recuperar los términos utilizados para identificar a los beneficiarios de los servicios sociales que comenzaron a instaurarse desde los años treinta y los sentidos que se le otorgaron.

\section{Las políticas sanitarias en los años 1930}

Córdoba, junto a las demás provincias de la región pampeana y las economías regionales como Mendoza, fue protagonista a partir aproximadamente de 1920 de la construcción de un esquema de salud y asistencia social de escala provincial. Desde esa época comenzó a conformarse una infraestructura sanitaria 
subnacional con la instalación de diversos establecimientos destinados a tratar las consideradas enfermedades sociales, como la sífilis, la tuberculosis y aquellas afecciones que elevaban los índices de mortalidad infantil. El eje estuvo puesto en los espacios urbanos, y la mayoría de esos dispositivos se localizaron en la ciudad capital y en las cabeceras departamentales. A partir de los años treinta, la administración provincial sufrió una transformación más extensa de sus instrumentos sanitarios, con la creación de infraestructura hospitalaria y de consulta médica curativa y generalista. Entre 1930 y 1943, fueron creados aproximadamente 58 dispensarios de primeros auxilios y 11 hospitales regionales distribuidos en diferentes localidades (Ortiz Bergia, 2015).

Esas nuevas políticas sociales en el espacio provincial fueron implementadas junto a un repertorio de definiciones sobre quiénes debían acceder a esos nuevos servicios. Un análisis de los términos utilizados para identificar a los beneficiarios da cuenta de un campo heterogéneo. En el área de la salud se escindieron dos categorías. En la dimensión preventiva de la salud, ligada a la lucha contra las enfermedades infectocontagiosas, se reglamentó una base universal de acceso irrestricto a los servicios de salud, la "población”. Esta categoría dotó al Estado de la capacidad para regular el comportamiento del conjunto de los individuos. La "población" era víctima de la peste bubónica, la viruela y, luego en la posguerra, objeto de las políticas de alimentación, deportes y controles sanitarios. A la prevalencia de este objeto de las políticas sanitarias subyació el proceso de gubernamentalización del Estado en el siglo XX, con la emergencia de una preocupación por los grupos humanos y el gobierno de sus condiciones de vida y de reproducción (Foucault, 2006). Hasta tiempos muy recientes las agencias sanitarias argentinas solo habían generado intervenciones que tenían como objeto las epidemias, la tuberculosis, las venéreas y la mortalidad infantil, áreas sobre las que se articulaba bien esa noción de "población".

Sin embargo, las nuevas políticas sanitarias organizadas en torno a la oferta de servicios curativos, como los dispensarios de primeros auxilios y los hospitales generalistas, cristalizaron un sujeto de atención diferente. Si seguimos los discursos de la época podemos tener una idea aproximada del mismo. Según la dirigencia cordobesa, "para el Estado es un deber la asistencia médica del enfermo pobre o de recursos limitados, los que tienen el derecho a exigir a los poderes públicos y a la sociedad lo humanamente remediable de su triste situación.”4 Es por eso que se afirmaba que "esta ayuda de la Sociedad no debe ni puede ser considerada como obra de caridad o beneficencia, sino como el simple cumplimiento de un deber, de una obligación o de una deuda de la sociedad entera para sus individuos que por cualquier razón circunstancial, no están en condiciones de valerse por sí mismos." Los enfermos "pobres", las personas de "escasos recursos" y quienes no pudieran "valerse por sí mismos" eran los legítimos beneficiarios de esas intervenciones. En los reglamentos redactados para los nuevos Dispensarios Provinciales de Primeros Auxilios y Profilaxis General, destinados a facilitar a los

4 Dell Acqua, Diario de Sesiones de la Cámara de Senadores de la Provincia de Córdoba, 1941, p. 886.

5 Zinny, Diario de Sesiones de la Cámara de Senadores de la Provincia de Córdoba, 1942, p. 480. 
habitantes de la campaña servicios de atención médica general sin cargo, los beneficiarios eran los "enfermos menesterosos", "enfermos desvalidos" y "pobres". Esos términos fueron reiterados en los reglamentos, memorias e informes. En 1933, al reglamentarse los dispensarios de primeros auxilios fue dispuesto que en los mismos fueran atendidos gratuitamente y lo mismo fuera hecho, pero a domicilio, con los "enfermos desvalidos cuyo estado no les permita trasladarse al consultorio", lo que diferenciaba la consulta gratuita de la paga. ${ }^{6}$ Para 1938, el gobierno reiteró esa disposición, pero volvió necesario especificar que las visitas domiciliarias eran para los "enfermos desvalidos y pobres".

En el nuevo reglamento de hospitales, la agencia sanitaria provincial dispuso que los mismos estuvieran "destinados a la hospitalización y curación de los enfermos pobres e indigentes". Los planes de edificación de servicios para enfermos de lepra fueron diseñados también para los "enfermos indigentes". En las memorias del Consejo de Higiene eran mencionados los "enfermos sin recursos", quienes "carecen de medios" (Provincia de Córdoba, 1940: 138) y las "clases menesterosas". 10 Para 1938, fue establecido que las enfermeras visitadoras de las gotas de leche debían realizar entrevistas domiciliarias con quienes según el registro civil tenían un recién nacido con la finalidad de brindarles instrucciones sobre la crianza infantil y "en los casos de personas pobres" también podían ofrecer los servicios de la cantina maternal, la gota de leche y el dispensario infantil. ${ }^{11}$

Así como los enfermos "pobres" eran los beneficiarios, quienes estaban excluidos de los servicios asistenciales provinciales eran los "pudientes". Por esos años, el gobierno aconsejó que el suero antidiftérico fuera solamente para las "personas realmente carentes de recursos" y no para quienes fueran "pudientes". ${ }^{12}$ Lo mismo fue establecido en el reglamento de los dispensarios, en donde era aclarado que "En caso de urgencia o accidente acaecidos a obreros con patrón responsable o asegurado, o a personas pudientes, los Dispensarios se reducirán a prestar los primeros auxilios, hasta tanto el enfermo o accidentado pueda ser trasladado para continuar su cura particularmente". ${ }^{13}$

6 Archivo de Gobierno de la Provincia de Córdoba (en adelante AGPC), Serie Gobierno, 1933, t. 13, f. 90r, dto. $29.679 \mathrm{~A}$.

7 Decreto 40.627/38 A. Archivo de Gobierno, Ministerio de Gobierno: Legislación sanitaria. Leyes y decretos vigentes en materia de sanidad y profilaxis general, 1944, p. 108.

8 Decreto 49.299/42 A. Archivo de Gobierno, Ministerio de Gobierno: Legislación sanitaria. Leyes y decretos vigentes en materia de sanidad y profilaxis general, 1944, p. 130.

$9 \mathrm{Si}$ seguimos un registro de las personas atendidas en los dispensarios provinciales por lepra las profesiones aparecidas predominantemente eran quehaceres domésticos, cocinera, jornalero, lavandera, empleados de correo, ferrocarriles, empleados y agente de policía. También aparecían un propietario, una profesora de idiomas y un chacarero (Garzón, 1934: p. 120).

10 Archivo de la Legislatura de Córdoba, Memoria del Ministerio de Gobierno, 1942, f. 57r.

11 Decreto 40.627/38 A. Archivo de Gobierno, Ministerio de Gobierno: Legislación sanitaria. Leyes y decretos vigentes en materia de sanidad y profilaxis general, 1944, p. 110.

12 AGPC, Gobierno, 1941, t. 35, f. 66r.

13 Decreto 4.575/41 A. Archivo de Gobierno, Ministerio de Gobierno: Legislación sanitaria. Leyes y decretos vigentes en materia de sanidad y profilaxis general, 1944, p. 112. 
La distinción entre enfermos "pobres” y "pudientes" constituía un lugar común en la jerga política. El gobernador Amadeo Sabattini, quien ejercía la práctica médica, explicaba que la provincia había incrementado los subsidios a los hospitales de beneficencia para asegurarse que sus prestaciones fueran absolutamente gratuitas, pero evitando "que los pudientes disfruten de sus servicios, desalojando a aquéllos que por sus condiciones económicas son sus destinatarios.”14 Para 1942, su sucesor en la gobernación explicaba a las Cámaras legislativas que la reducción en la atención de los dispensarios provinciales en parte era producto de que los mismos "se han limitado a los enfermos realmente pobres, tratando de evitar toda infiltración de personas pudientes."15

De tal modo, "pobre" y "pudiente" fueron pares de adjetivos antagónicos destinados a clasificar y calificar a los enfermos dentro de ese sistema de salud. ${ }^{16}$ Pero no fue exclusivo de los cordobeses el gusto por esa escisión. Un recorrido por otras jurisdicciones también muestra la aparición de esas categorías como mecanismos de delimitación de las clientelas de los servicios estatales.

Para acceder al Open Door de Oliva, la Comisión Nacional de Asilos y Hospitales Regionales exigía "sumaria información" hecha ante Juez competente a efectos "de probar su extrema pobreza" y asegurar la gratuidad del servicio. ${ }^{17} \mathrm{El}$ primer presidente de esa comisión, al inaugurar los servicios hospitalarios generales de Bell Ville, identificaría a los "enfermos indigentes" y los "enfermos menesterosos" como sus destinatarios. ${ }^{18}$ En los territorios nacionales algunos estudios locales también destacan la existencia de un registro de pobres y de los controles instaurados para asegurar un uso restrictivo de los servicios estatales. ${ }^{19}$ Algo semejante sucedía en otras jurisdicciones provinciales. En la prensa mendocina era publicada la estadística de la Inspección de Sanidad y la cantidad de certificados de pobreza expedidos ${ }^{20}$ y las autoridades explicaban que la vacunación de los niños contra la difteria sería gratuita para los "pobres de solemnidad", quienes debían enviar a sus hijos a la Asistencia Pública y a los hospitales provinciales; no así en el caso de "los pudientes", quienes debían vacunarse con "sus médicos particulares". ${ }^{21} \mathrm{El}$ decreto reglamentario de los dispensarios dependientes de la Dirección General de Higiene de la provincia de Santa Fe establecía en su primer artículo que los médicos a cargo "Fijarán horario de dos horas diarias para la asistencia de las personas pobres, las que deberán presentar certificados de tales otorgados por la

14 (1 de febrero de 1940), Los Principios, Córdoba, p. 10.

15 Mensaje a la legislatura, Del Castillo, 1942, p. 23.

16 Bourdieu (1998: 479). Sobre el tema de los cierres sociales a través del uso de pares categoriales, ver: Tilly (2000).

17 AGPC, Gobierno, 1933, t. 4, f. 411r.

18 En ocasión de dar un discurso sobre el Hospital de Antituberculosos de Santa María, Cabred daría cuenta de la identidad común entre "pobre" y "obrero" al afirmar que "es necesario instalar, más o menos, 63.000 camas, cuya tercera parte, 21.000 sería para tuberculosos pobres, pues la población del país constituye un tercio de la población total." Cabred (s/f: 47, 49, 117).

19 Sobre el funcionamiento de un registro de pobres municipal, ver: Infeld (2009).

20 (5 de mayo de 1930), “En los hospitales y en la Asistencia Pública”, Los Andes, Mendoza, p. 5.

21 (10 de mayo de 1930), “Es considerable el número de casos de difteria que se producen diariamente en esta ciudad y departamentos", Los Andes, Mendoza, p. 5. 
Policía, Jueces de Paz o Comisiones de Fomento". Quienes eran "pobres de solemnidad" también accedían a los medicamentos (Provincia de Santa Fe. Dirección General de Higiene, 1933:10, 23). Cinco años después, los dispensarios seguían en funciones prestando su atención "a los enfermos pobres de las regiones donde están ubicados” (Provincia de Santa Fe. Dirección General de Higiene, 1938: 17). Para 1943, el ministro de Salud de esa provincia, Irigoyen Freyre, inauguraba servicios hospitalarios que asegurarían prestaciones gratuitas "por entender que las necesidades más apremiantes en el orden médico-curativo las experimenta la clase desposeída de recursos económicos”. ${ }^{22}$ En la Asistencia Pública de la ciudad de Buenos Aires, identificada tradicionalmente por su liberalidad en la atención sanitaria, el certificado de pobreza fue restituido en $1942 .{ }^{23}$

En los años treinta, esos mecanismos de segmentación de la clientela no fueron necesariamente asociados con dispositivos tradicionales de gestión de los problemas sociales. En Uruguay, la modernización de su sistema de salud incluyó la eliminación del sistema de "puertas abiertas" y la creación de un departamento de admisión de enfermos destinado a "controlar que los servicios del Estado vayan exclusivamente, como la ley lo requiere, a beneficiar a las clases necesitadas del país, y no se produzca la antes frecuente filtración de elementos pudientes que, aprovechando las liberalidades de nuestra asistencia estatal, sustraen al menesteroso lo que, por su calidad de tal le corresponde.” Por ese medio, se lograba amparar al enfermo, al Estado y a la corporación médica. Eso imponía dos clases de asistencia: 1. "la gratuita para los indigentes o carentes de recursos suficientes. 2. La renumerada, para los débiles económicos y en determinadas condiciones, para los de solvencia económica notoria." Esas diferencias eran establecidas mediante "severos controles" en las solicitudes. Los enfermos del país vecino para gestionar su carnet debían presentar una declaración jurada económica y familiar revisada por inspectores y fiscalizadores (República Oriental del Uruguay, 1942: 9, 390). Según la memoria oficial: "La República Oriental del Uruguay tiene el orgullo de ser la primera nación de América que ha instalado este sistema, en su todo de acuerdo con la reconocida envergadura de su legislación social y con los progresos que en materia de asistencia, la colocan entre los primeros países del mundo." (República Oriental del Uruguay, 1942: 396).

Ahora bien, la utilización de las etiquetas de "pobre" y "pudiente" fue objeto de confrontaciones producto de su capacidad para pensar a la sociedad, delimitarla, restringirla y prescribir comportamientos. Al interior de la profesión médica y los partidos políticos existieron voces que bregaron por la socialización de la medicina y el acceso irrestricto a los servicios de salud. Así pues, en los hospitales porteños, rosarinos y mendocinos tenemos constancia de coyunturas en las que se eliminaron esas clasificaciones en procura de reducir los procedimientos restrictivos y estigmatizantes que los mismos suponían. En

22 (15 de mayo de 1943), "Hoy han sido inaugurados los hospitales de Santa Rosa y Cayastá (Garay)", El Litoral, Santa Fe, p. 5.

23 (29 de mayo de 1942), "Cree improcedente el arancel hospitalario el Colegio de Médicos", El Mundo, Buenos Aires, p. 13; (23 de mayo de 1942), "El arancel hospitalario, además de no solucionar nada, atenta contra la salud de la población pobre", Crítica, Buenos Aires, p. 3. 
el caso cordobés, ese tipo de intervenciones críticas fue más bien coyuntural y no eliminaron la tendencia a asociar los servicios públicos con la población de escasos recursos.

\section{¿Quién es el enfermo pobre?}

La categoría de enfermo "pobre" tiene un largo recorrido y su sentido no necesariamente ha sido el mismo a través del tiempo. Quienes estudian los dispositivos hospitalarios coloniales sostienen que los mismos tenían como finalidad alojar a los pobres (Quesada, 1917) y estaban erigidos "más sobre una cierta concepción de la pobreza que sobre una real estrategia sanitaria” (Alzate Echeverri, 2008: 205). Esto implicaba que los médicos eran raros y hasta avanzado el siglo XVIII las prácticas hospitalarias estuvieron escasamente medicalizadas; los hospitales recibían a los enfermos y a quienes no lo eran, en pos de asegurar su cuidado y consuelo (Alzate Echeverri, 2003). En otras palabras, hasta el siglo XIX los hospitales no realizaron distinciones claras entre "enfermo" y "pobre" y sus funciones asistenciales y de salud (Fernández, 2017: 25).

Durante el período de gobiernos liberales, los hospitales siguieron siendo los lugares de atención de los enfermos "pobres", pero ahora en esos establecimientos no solo concurrían por asilo, sino por una real posibilidad de curación a instancia de los avances médicos. Por esa época, en muchas provincias los principales oferentes de servicios asistenciales eran los hospitales de beneficencia y sus destinatarios eran los "enfermos menesterosos", ${ }^{24}$ los "necesitados", ${ }^{25}$ quienes estuvieran en condiciones de probar su "estado de pobreza". ${ }^{26}$ Subyacía a estas categorías una ideología de la pobreza por la que los pobres eran identificados por su incapacidad moral o material para el trabajo, desposeídos de responsabilidad y de capacidad para modificar su situación. Esto significaba entender a la pobreza como un problema de incapacidad individual y no una consecuencia de las desigualdades económicas y sociales (Moreyra, 2009).

En ese contexto, comenzó a estructurarse la infraestructura de servicios públicos analizada previamente, pero los principios sobre los que se instituyó la función estatal fueron diferentes a los del modelo benéfico asistencial. Esto llevó a una readecuación de la categoría de "pobre" a la que se intentó dotar de nuevos sentidos. Los enfermos "pobres" en los discursos estatales fueron distanciados de la noción de "pobre válido" e "inválido". La categoría de enfermo "pobre" como beneficiario de los servicios sociales públicos comenzó a ser un equivalente de "clases humildes", "clase trabajadora", "clase proletaria", "obreros", "trabajadores y empleados".

24 Solicitud del Hospital San Roque, AGPC, Gobierno, 1936, t. 40, f. 2 r.

25 Solicitud del Hospital Español de la Sociedad de Beneficencia Española, AGPC, Gobierno, 1937, t. 13, f. $388 \mathrm{r}$.

26 Reglamento del Hospital Tránsito Cáceres de Allende, AGPC, Gobierno, 1941, t. 39, f. 47r. 
Para algunos contemporáneos, la igualación entre enfermos "pobres" y "trabajadores" había sido producida por la crisis económica, porque "nunca, como en el tiempo presente, la miseria se cebo tanto con el desheredado que, privándole de trabajo y de salario, lo ha colmado de enfermedades físicas y morales, tal vez porque el ambiente de pobreza que ha engendrado esta crisis se ha amalgamado en todos los cuerpos y en todos los espíritus." ${ }^{27}$ En esos términos, se entiende que en ocasiones el sujeto de la asistencia social fuera pensado desde la lógica del contribuyente y el sujeto de derecho fuera la sociedad de productores. Al tratarse el proyecto de construcción de un nuevo hospital en la ciudad, un legislador señalaba que "el gobierno de Córdoba debe dar a su pueblo la obra que realmente merece; la asistencia social no puede ser realizada por caridad, el enfermo tiene el derecho a exigir que se le atienda porque es contribuyente del Estado en que vive, y el Estado tiene la obligación de proporcionar el servicio que ese enfermo requiere." ${ }^{28}$ Desde la retórica estatal, el acceso de los trabajadores a los servicios sociales se sustentaba en una noción de justicia conmutativa, quienes contribuían con su trabajo, sus impuestos y su actividad social eran ciudadanos de pleno derecho y como tales merecían beneficiarse de la producción colectiva.

Qué implicaba esta noción de acceso al bienestar estatal. Esa categoría suponía una determinada relación con el Estado y con el resto de la sociedad. Las personas no accedían al bienestar por su simple pertenencia social tal cual era definida pocos años después por Marshall. En cambio, la acción estatal sobre un sector de la sociedad era "justa" y "humanitaria", en la medida en que retribuía el valor social de los individuos trabajadores y moralmente responsables. Si bien este sujeto de derecho se alejaba de la idea de ciudadanía desde la lógica de pertenencia social universalizante, el uso del término contribuyente suponía una novedad respecto de las definiciones de beneficiario construidas a partir relaciones de paternalismo o caridad. La lógica del contribuyente como fuente de legitimidad de las políticas sociales no constituyó una novedad de los años treinta. Los dirigentes políticos contaban con modelos de diversas políticas sociales como la educación, construida bajo el imperativo político de formar sujetos que a través de los roles que cumplirían en el porvenir, como ciudadanos y soldados, retribuirían el esfuerzo colectivo realizado por ellos en un tiempo presente.

En definitiva, el tradicional destinatario de la beneficencia pública, caracterizado por la indigencia y definido por su invalidez, moral y material, fue paulatinamente modificado para incluir también a los trabajadores e, incluso, a quienes comenzaban a distinguirse como sectores medios (Ortiz Bergia, 2009: 120). Pero no solo los políticos se involucraron en la definición del sentido del binomio enfermo pobre/pudiente. En el sostenimiento de esa frontera fueron los médicos quienes negociaron intensamente cuál debía ser el destinatario de la medicina oficial. ${ }^{29}$ 
A mediados de los años treinta, en una conferencia sobre la "crisis de la profesión médica”, Antonio Cetrángolo, un reconocido tisiólogo cordobés, al referirse a la población de los hospitales públicos, los identificaba como "gente del pueblo", "que no tiene como pagarse un buen cirujano" (1935: 544). También criticaba el mal uso de los hospitales por quienes no eran enfermos "pobres", los "pobres diablos", si no "los que más pueden", los que en general eran recomendados por políticos influyentes (1935: 547-552). De su testimonio se desprende el sentido dado a la categoría "pobre" en contraposición a la de "pudiente". El enfermo "pobre" es aquel que no puede pagarle al médico, el "pudiente" es su potencial cliente. Pares definidos por sus posibilidades para financiar o no la atención médica privada.

La puja entre los médicos y los funcionarios estatales en la definición de categorías de beneficiario de los servicios médicos públicos debe comprenderse en el marco de las transformaciones que estaba sufriendo la práctica médica. Desde finales del siglo XIX, mientras los enfermos de estratos bajos eran atendidos en el hospital, los otros, los sectores medios y altos, recibían cuidados en sus propias viviendas. Pero estas prácticas comenzaron a cambiar durante la entreguerras. La aplicación de nuevos tipos de análisis, la lenta implementación de los mecanismos de asepsia y antisepsia y el desarrollo de una compleja aparatología de diagnóstico y terapia como los rayos $\mathrm{X}$, fueron consolidados como dispositivos terapéuticos y de análisis. Las novedades aplicadas a la medicina tuvieron consecuencias en la generación de mayores expectativas en su eficacia y en la consolidación de una estructura de prestación médica centrada en el hospital. Los establecimientos hospitalarios fueron desde entonces convertidos en lugares preferentes de prestación que permitían el acceso a equipamientos de complejidad y a las nuevas especialidades médicas. ${ }^{30}$ Esa conversión del hospital en el dispositivo de prestación de servicios de salud moderno que conocemos hoy modificó las lógicas de la demanda y puso en crisis la práctica privada de los médicos. ${ }^{31}$ En el marco de este proceso, el crecimiento en el número de personas que podían ser identificadas como "pobres" fue paralelo al incremento en los costos del cuidado individual de la salud, producto de la necesidad de consultar con varios especialistas, utilizar numerosos instrumentos de diagnóstico y tratamiento, internarse en modernos hospitales y consumir medicamentos específicos.

Una encuesta realizada por esa época había arrojado que de quince médicos solo cinco de ellos lograban subsistir por medio de su actividad particular, y los restantes debían equilibrar sus ingresos con distintos puestos públicos, privados y rentas propias. De estos datos, el autor del artículo concluía que más que un problema

30 Esto último se puso en evidencia en la creciente organización de sociedades profesionales como las sociedades de tisiología, medicina interna, pediatría, oftalmología, dermatología, biología, radiología, cirugía y otorrinolaringología.

31 Es posible enlazar la demanda médica de restricción de la oferta pública con la expansión del subsistema privado de servicios de salud durante las décadas siguientes. En 1943 en las fuentes oficiales se hacía referencia a 4 sanatorios privados en la ciudad de Córdoba, con aproximadamente 40 y 30 camas cada uno, y 17 en el resto de la provincia incluyendo algunos especializados en el tratamiento de la tuberculosis. Para 1951 hemos constatado la existencia de aproximadamente 22 sanatorios privados solamente en la ciudad de Córdoba y dos hospitales obreros, el de los ferroviarios y el de los bancarios. Estos nuevos establecimientos y su dispersión en el territorio provincial habría sido la respuesta de la corporación médica a la necesidad de satisfacer la demanda de atención hospitalaria por parte de los sectores con poder adquisitivo, delimitando también una clientela para los servicios hospitalarios públicos (Ortiz Bergia, 2015). 
de exceso de médicos, el mismo era de demanda de atención, dado que más allá de la "mejora progresiva" de la salud pública, los enfermos eran "acaparados cada día más" por los servicios estatales, empresas de seguros, mutualidades y socorros mutuos. Uno de los problemas de los médicos era la "asistencia hospitalaria sin restricción”, junto a las empresas médicas y los practicantes de la medicina sin título habilitante (Herrero y Prece, 1935). En ese contexto, fue vital para la profesión intervenir delimitando ¿quiénes debían acceder a la medicina hospitalaria pública? ¿todos los ciudadanos, todos los contribuyentes o solo los que no podían financiar la medicina privada? La decisión respecto a en donde dibujar la frontera entre enfermos "pobres", acreedores de servicios médicos gratuitos, y "pudientes", obligados a su erogación, fue neurálgica en las luchas del gremialismo médico.

A raíz de los diagnósticos críticos sobre la situación de la profesión, el Círculo Médico cordobés nombró una comisión destinada a estudiar la solución al "malestar médico". Los profesionales presentaron un proyecto de reglamentación hospitalaria cuya finalidad fue regular el acceso de la población a los servicios estatales, en especial intentando restringir la atención de "los enfermos pudientes", quienes eran susceptibles de pagar una consulta privada. ${ }^{32} \mathrm{El}$ proyecto elaborado regulaba la atención de los hospitales públicos e, incluso, de las mutualidades, intentando que los mismos redujeran su clientela a quienes carecieran de capacidad de pago y proponían procedimientos exhaustivos para fijar ese estado. En los casos en que quienes tuvieran que hacer uso de los hospitales no fueran enfermos "pobres", eran necesarias tarifas semejantes a los consultorios privados. ${ }^{33}$ Para los médicos, quien debía hacerse atender en los hospitales, era "quién no puede pagar ni poco ni mucho." 34

Esta discusión sobre la definición de los beneficiarios de los nuevos servicios estatales tampoco pareció ser excluyente de los médicos cordobeses. La revista del Colegio de Médicos publicó una serie de textos producidos por médicos mendocinos en donde, frente a la falta del certificado de pobreza como procedimiento administrativo de delimitación de pacientes en los servicios provinciales, solicitaban que fuera una atribución de los médicos oficiales la aplicación de los criterios de selección entre los "pobres" y los "pudientes" y que fuera instaurada una ficha económica de la población, en reemplazo de la antigua certificación. ${ }^{35}$ Algo semejante demandaban sus colegas bonaerenses. El directorio del Colegio de Médicos de la Provincia de Buenos Aires solicitaba al nuevo director de higiene la reglamentación de los servicios provinciales "para que en esos establecimientos sólo sean atendidas las personas de escasos recursos y se evite lo que ya es una práctica en los mismos, es decir, que ellos se utilicen por personas de reconocida solvencia y en condiciones de recurrir a los servicios particulares." ${ }^{36}$

32 Diario de Sesiones de la Cámara de Senadores de la Provincia de Córdoba, 1936, p. 143.

33 Diario de Sesiones de la Cámara de Senadores de la Provincia de Córdoba, 1936, pp. 621-3.

34 AGPC, Gobierno, 1942, t. 46, f. 193r.

35 (Enero de 1941), "Solicitan de la dirección del Hospital se ponga coto a los abusos de enfermos pudientes", Revista del Colegio Médico de Córdoba, núm. 11, pp. 13-17.

36 (12 de mayo de 1940), “El colegio de médicos reitera una reclamación”, La Nación, Buenos Aires, p. 13. 
La solución al "malestar médico", a esta crisis de la consulta privada, parecía ser la delimitación de las clientelas de cada circuito o la adopción de medidas que aseguraran la socialización de la medicina. Más precisamente, parecía existir consenso en que la atención hospitalaria gratuita debía ser para los enfermos "pobres", etiqueta que identificaba a quienes no podían abonar esos servicios, en contraposición a los "pudientes". La dirigencia política local no era ajena a este consenso en un contexto institucional y financiero en que las posibilidades de alcanzar una efectiva socialización de la medicina eran muy escasas.

\title{
Procedimientos administrativos y clasificaciones
}

\author{
"Para que los carentes de recursos puedan ser atendidos \\ en un hospital deberán jurar que son pobres o proveerse \\ de un certificado de pobreza. El enfermo debe pasar por la \\ humillación de patentar su pobreza: ir primero a la policía, \\ tenga o no fuerzas antes que al hospital. Con este certificado \\ hay que ir humildemente al hospital, esperar con la cabeza baja \\ que sean atendidos los pagan para obtener después de ellos \\ una migaja de asistencia social [...] No hay humillación en ser \\ pobre; pero, no es posible colocar al hombre en la situación de \\ los leprosos de la edad media, los cuales, mediante una matraca \\ anunciaban desde lejos su presencia." ${ }^{37}$
}

El beneficiario de las políticas sanitarias públicas fue entonces el enfermo "pobre", "humilde", "carente de recursos", adjetivos que intentaban identificar a quienes no podían financiar la medicina privada. Dentro de la categoría de enfermo "pobre" entonces podían incluirse un conjunto muy heterogéneo de sujetos sociales. Muchas de las categorías que fundamentaron las nuevas políticas sociales fueron mantenidas como términos opacos, difíciles de definir y delimitar; justamente, su principal valor fue esa escasa claridad y consecuente flexibilidad. Términos como los de enfermo "pobre" y "desheredado", se caracterizaron por su enorme imprecisión, lo que nos lleva a interrogarnos sobre cómo y quiénes fueron entonces los encargados de definir y aplicar esas clasificaciones.

El límite entre quienes eran enfermos "pobres" o "pudientes" fue establecido en su capacidad para acceder al mercado de salud y, en consecuencia, fue habitual que la delimitación entre ambos fuera discutida en términos de ingresos económicos. En los proyectos presentados en la Legislatura cordobesa, destinados a regular el acceso a los establecimientos de beneficencia, la población beneficiaria fue definida por un nivel de ingresos común a la mayoría de los trabajadores. En distintas propuestas, ninguna de ellas sancionada, fueron presentados límites que rondaban los 160, 200 y 300 pesos en el caso de las prestaciones médicas más costosas, como las operaciones quirúrgicas, inalcanzables incluso para los sectores

37 (6 de junio de 1942), "La Cámara de Diputados se ocupará esta semana de los aranceles hospitalarios", Crítica, Buenos Aires, p. 5. 
medios. ${ }^{38}$ Algo similar sucedió a nivel municipal. Según las ordenanzas en vigencia, podían acceder a los servicios individuos con sueldos inferiores o superiores a 150 pesos, que era un nivel de ingresos bastante común entre los asalariados. Entre los sujetos excluidos, salvo casos de emergencia, estaban quienes tuvieran negocios de cierta envergadura, los que trabajaban en reparticiones públicas con consultorios y farmacias anexas, y los que pertenecían a alguna mutual. Una disposición similar propuso la corporación médica, preocupada por delimitar en forma rigurosa quiénes debían ser considerados enfermos "pobres” y susceptibles de acceder a los servicios gratuitos. En los hospitales estatales esto sería constatado a través de un fichero cuya exhaustividad tenía una intencionalidad disuasoria: se formaría con el nivel del salario, certificado de pobreza, estado civil, carga familiar e impuestos. ${ }^{39}$ Lo interesante del uso de los ingresos para definir al beneficiario de los servicios públicos es que demuestra que la etiqueta de "pobre" quedaba yuxtapuesta a otras como trabajadores, obreros, sectores medios e incluyéndolos uniformemente por su incapacidad para abonar los costos de las prestaciones médicas.

Sin embargo, lo que se desprende de las normativas aplicadas y de la vida cotidiana de las reparticiones estatales es que la instrumentación de este procedimiento para delimitar el acceso a los servicios públicos no fue una práctica tan habitual. Lo más generalizado, en cambio, fue dejar la atribución de certificar el "estado de necesidad" en personas "merecedoras de confianza", quienes debían testimoniar esa condición. La declaración de pobreza de origen colonial fue reactualizada con la finalidad de restringir a un sector de la población del acceso a los nuevos servicios estatales. ${ }^{40} \mathrm{En}$ la vida cotidiana de las agencias estatales, el tradicional certificado operó para habilitar el uso de los comedores escolares, las colonias vacacionales, las becas en los conservatorios de música y en los servicios de salud. ${ }^{41}$ En el caso de los hospitales provinciales, fue establecido que los enfermos debían “certificar su estado de pobreza cuando la Dirección lo creyera conveniente". ${ }^{42}$ En el caso del hospital universitario, fue reglamentado que toda persona recibiría atención gratuita si expresaba verbalmente que no tenía recursos y llenaba una declaración. Si se daba el caso de comprobarse que la declaración era falsa, se procedería a embargar sus bienes por el doble de los servicios prestados. ${ }^{43}$

Es necesario reconocer que la efectividad del certificado de pobreza descansaba sobre dos procedimientos de segmentación de los beneficiarios. Inicialmente, los agentes estatales responsables de certificar la condición de pobreza debían

38 Diario de Sesiones de la Cámara de Senadores de la Provincia de Córdoba, 1936, pp. 621-3; Diario de Sesiones de la Cámara de Diputados de la Provincia de Córdoba, 1939, p. 212.

39 Diario de Sesiones de la Cámara de Senadores de la Provincia de Córdoba, 1936, pp. 621-3.

40 Para un análisis del certificado de pobreza en los siglos XVIII y XIX, ver: Parolo, 2005-2006; Rebagliati, 2016.

41 Sobre la utilización del certificado de pobre en el acceso a los servicios de salud para esta época también hay trabajos sobre el caso de Comodoro Rivadavia. Infeld (2009).

42 Archivo de Gobierno, Ministerio de Gobierno: Legislación sanitaria. Leyes y decretos vigentes en materia de sanidad y profilaxis general, 1944, p. 136.

43 (2 de julio de 1946), "Se dispuso suprimir los aranceles que se cobraban en los servicios hospitalarios de la Universidad", La Voz del Interior, Córdoba, p. 9. 
ponderar testimonios e indicadores materiales y simbólicos, como la vivienda, las ocupaciones, los usos del tiempo libre, las formas de crianza, la vestimenta, las formas de hablar y de comportarse; paso seguido, debían investir a la persona que solicitaba el servicio o el certificado de la condición de legítimo beneficiario de los servicios sociales dada su condición de "pobre". No es necesario ahondar en las críticas permanentes que suscitó la labor llevada a cabo por dirigentes políticos, policías, médicos y otros agentes, acusados de hacer un uso discrecional de esta capacidad de asignar "dones".

El segundo procedimiento sobre el que descansó el certificado de pobreza fue el de la autopercepción, cómo los sujetos se apreciaban y cómo querían presentarse a la sociedad. Esto remite necesariamente a los ajustes que una persona debe realizar para adecuarse a las nociones que tiene de sí misma y que espera que los otros compartan (Goffman, 2006: 144). Si bien no es posible asumir que los sentidos dados al certificado de pobreza fueran los mismos entre los agentes estatales y quienes se pensaron susceptibles de ser etiquetados como enfermos "pobres"; 44 de todos modos, para tratar con el Estado, para acceder a sus oficinas y para beneficiarse de sus bienes, las personas debieron ajustar su presentación a las categorías estatales en vigencia, adecuar sus identidades y sus prácticas a lo que era esperado de quienes estaban en condiciones de beneficiarse de las políticas sociales. En consecuencia, para usufructuar los servicios de salud era necesario reconocer la condición de pobreza, la carencia e incapacidad para sufragar los costos médicos y exteriorizar signos inequívocos de esa condición. La persona debía reconocerse enfermo "pobre" y representar ese rol en la forma de vivir, vestir y comportarse. En muchos de los documentos producidos por los mismos beneficiarios del bienestar estatal ronda habitualmente la noción de "merecimiento", indicio de que sobre ellos recaía la tarea de demostrar su condición de legítimos destinatarios del bienestar estatal. ${ }^{45}$

El procedimiento de gestionar el certificado de pobreza fue instituido entonces como un ritual, el certificado era una marca que asignaba atributos de "pobre" o "carentes de recursos" a los individuos frente a la sociedad y al Estado. Para algunos esta constituía una tarea sensible. Un jefe policial decidió que la nómina de beneficiarios de los certificados de pobreza fuera publicada en la prensa diaria para evitar que se aprovechasen quienes no debían, los "pudientes". En los listados no solo aparecía el titular del certificado, sino también la institución para la cual se concedía: la Asistencia Pública y los dispensarios municipales, las gotas de leche provinciales, el hospital universitario del Clínicas, el hospital de la colectividad española y el hospital de niños dependiente de la Sociedad de Beneficencia. ${ }^{46}$

44 Aquí nos apoyamos en las discusiones desarrolladas por Bourdieu, 1985: 12.

45 La condición de merecimiento constituyó un elemento característico de las relaciones sociales que sustentaban la asistencia benéfica. Golbert (2008: 21); Lorenzo del Río (2011: 212).

46 (29 de agosto de 1943), "Los certificados de pobreza expedidos en las seccionales”, La Voz del Interior, Córdoba, p. 11. 


\title{
¿Quién quiere ser un enfermo pobre?: marcas, estigmas y diferenciación social
}

\author{
“...al hacerle revisar por médicos especialistas en \\ esa enfermedad me han dicho que debe operarse \\ imprescindiblemente, pero cobran uno 1000 y otro 1500 por la \\ operación, y forzosamente debo de desistir en hacerla operar \\ particularmente, como ella lo hubiera merecido en premio a \\ los sacrificios que ha hecho y hará aún por los menores, pero \\ mi pobre hermana tendrá que operarse en un hospital por no \\ haber otra solución." \\ Carta a Eva Perón de Fidel L. \\ AGPC, Gobierno, 1949, t. 8, s/f, Exp. 78-L-1949
}

Los trabajos de clasificación social condensados en el certificado de pobreza no fueron invariables. No debió ser lo mismo atenderse en un dispensario rural, en pueblos de menos de mil habitantes, que en los grandes nosocomios urbanos. No fue lo mismo acceder a la comisaría local, que a la central de policía. Asumir el adjetivo de "pobre" debió implicar cosas distintas en distintos lugares. Ahora bien, resulta dificultoso ponderar cómo la sociedad calificaba esa categoría y qué significado tenía adoptarla entre quienes debieron hacerse atender en los servicios de salud. Las clasificaciones existen como instrumentos estructurantes de prácticas sociales, sin embargo, que las mismas se impongan no quiere decir que sean asumidas invariablemente de manera unívoca. En este terreno solo podemos manejarnos con supuestos tentativos.

Podemos comenzar afirmando que la autoidentificación como "pobre" entre los sectores trabajadores era recurrente en los testimonios que aparecían en la prensa y en los archivos oficiales en situaciones vinculadas al consumo de bienes sanitarios. Esto último habilita a interrogarse sobre la manera en que esas clasificaciones sociales no solo fueron impuestas desde arriba, sino también utilizadas estratégicamente desde abajo en la generación de ventajas sociales en el usufructo de bienes públicos. Múltiples testimonios evidencian esa inscripción simbólica en el espacio social. Un obrero rural de Hernando manifestaba sobre una campaña en provecho del dispensario provincial llevada a cabo por el partido socialista local: "Considero que los trabajadores de todos los gremios deben colaborar con «La Voz Obrera» ya que los principales beneficiados seremos nosotros los pobres." Para un carpintero de la ciudad de Córdoba, los hospitales de beneficencia con subvenciones oficiales eran los lugares en que "el público menesteroso debe ser atendido gratuitamente no cobrándosele su asistencia médica [...] los pobres encontremos la asistencia médica gratuita que necesitamos para nosotros y para nuestros hijos." ${ }^{48}$ La autoidentificación como "pobre" parecía una acción necesaria que legitimaba los reclamos de asistencia y el usufructo potencial de un haz de derechos sociales que los funcionarios estatales no podían eludir sin consecuencias morales y políticas (Lorenzo del Río, 2011). 
Algo similar podemos aseverar respecto a su par "pudiente", utilizado para negar derechos a los servicios públicos. Los habitantes de un barrio cordobés intentaban argumentar en contra del traslado de un dispensario municipal a otra barriada porque la misma tenía numerosos beneficios y pobladores sin derecho a la asistencia. Según estos vecinos, el barrio Las Flores "tiene red telefónica, subcomisaría, médico residente que además lo es de la Asistencia Pública; buen servicio de ómnibus; una distancia de dos kilómetros de la Asistencia Pública; la mitad de su población es gente pudiente, y es menor la densidad de la misma que la de Villa Forestieri y Barrio Comercial." ${ }^{49}$

La utilización del adjetivo "pobre" y la solicitud de atención médica gratuita requerían reconocer las carencias económicas personales y la incapacidad para valerse por sí mismo, lo que implicaba costos en términos de valoración social. Esto parece desprenderse de la cita incorporada al inicio de este apartado. También se entiende de dos guiones de películas de la época en donde la atención en un hospital público era justamente un indicador de una situación destituyente, poco deseable para sujetos identificados con la clase media. Tanto en Kilómetro 111 $(1938)^{50}$ como en El Hincha (1951) la trama avanza con episodios en que distintos personajes intentan financiar la internación de sus familiares en sanatorios privados a pesar de los desajustes económicos que esto podía generar. En la boca de una de las actrices del film de Romero, "para qué están los hospitales" si no era para atender a quienes no tenían recursos. ${ }^{51}$

No es posible minimizar la carga estigmatizante que podía acarrear la etiqueta de "pobre" en la cultura popular y su capacidad como mecanismo de distinción social. Si parte de los mecanismos de diferenciación social que la clase media erigió respecto de las clases populares fueron cierres simbólicos construidos sobre la ética del trabajo, el esfuerzo familiar y la decencia moral, la noción de responsabilidad individual fue entonces un rasgo valorado socialmente que asignó un estatus negativo a quienes debían solicitar ayuda social (Graves, 2009).

49 (13 de octubre de 1943), "El dispensario de V. Forestieri", Los Principios, Córdoba, p. 4.

50 En la trama de esta película aparece un chacarero que se rehúsa a hacer operar a su hijo en un hospital. Kilómetro 111 (1938), dirección Mario Soffici, minuto 00:50:00. https://www.youtube.com/ watch?v=f8xYWbnyJOg (acc. 10/16).

51 El "Ñato", el protagonista de El Hincha (1951), debe vender un terreno comprado en cuotas en pos de pagar los servicios de un sanatorio para su madre. El Hincha (1951), dirección Manuel Romero, 1:17:00. https://www.youtube.com/watch?v=0jDWRIjm1Js. (acc. 10/16). 


\title{
Los enfermos pobres y la democratización del bienestar
}

\author{
"El Estado debe afrontar la asistencia médica integral en \\ beneficio de aquellos que ganan menos, será conveniente \\ semi-oficializar la medicina, respetando el libre ejercicio de \\ la profesión y la libre elección del médico por el enfermo y \\ fomentar por otra parte, para las clases pudientes el desarrollo \\ del régimen medicinal privado."
}

Presidente Juan Domingo Perón, apertura de Sesiones Legislativas, $1946^{52}$

Al ritmo del Estado nacional (Ramacciotti, 2009), entre los años cuarenta y cincuenta, se produjo un verdadero quiebre en las dimensiones de la infraestructura sanitaria cordobesa. Las decenas de dispositivos existentes en 1943 fueron convertidos, en 1954, en 168 dispensarios y salas de primeros auxilios y en 107 hospitales, estos últimos en su mayoría pequeños servicios con algunas salas anexas. Ese sistema de salud fue orientado a la atención gratuita curativa y generalista de la población de la jurisdicción y sustentó el sistema de salud público subsistente hasta la actualidad.

El ascenso del peronismo y las transformaciones operadas en las políticas sociales a partir de ese momento, nos lleva a interrogarnos sobre las modificaciones introducidas en las clasificaciones estatales y en la capacidad de designación que mantuvieron etiquetas como la de "pobre" y "pudiente" para identificar a los beneficiarios de la asistencia social pública. Al respecto, es posible afirmar que en ese contexto maduró más extensamente la distinción entre la noción de "población" ligada a la medicina social y sanitaria y la noción de enfermo "pobre" como aquel a quien estaba destinada la medicina asistencial del Estado. Como expresión de ello, en el Plan trianual cordobés fue establecido que la medicina asistencial debía ser gratuita y orientada a quienes no contaran con recursos suficientes. ${ }^{53}$ Tres años después, las autoridades sanitarias elaboraban un decreto en donde explicitaban que las cooperadoras de los establecimientos sanitarios provinciales debían colaborar para que "se preste a las personas carentes de recursos, asistencia médica amplia y completa, tratando de proporcionarle los elementos necesarios para su tratamiento." ${ }^{54}$ Descle esa lógica, las agencias estatales intervinieron en algunos hospitales de beneficencia con el fundamento de que en los mismos era necesario "propender al mejoramiento y organización de todos los servicios hospitalarios de la provincia, para que los servicios sean efectivos y lleguen a las clases menesterosas sin ninguna distinción." 55

El análisis exhaustivo de la documentación generada durante el período peronista por las agencias estatales permite constatar la subsistencia de adjetivos

52 (27 de junio de 1946), Los Principios, p. 1.

53 Diario de Sesiones de la Cámara de Senadores de la Provincia de Córdoba, 1952, p. 1215.

54 AGPC, Gobierno, 1955, t. 58, s/f, dto. 4223/d.

55 AGPC, Gobierno, 1949, t. 25, Exp. 62.166/49, f. 2. 
destinados a focalizar los servicios de salud. De manera más precisa, "pobres", "carentes de recursos", "menesterosos", "necesitados", "desheredados" y "humildes" son algunos de los términos que hemos recuperado en un análisis de contenido de las disposiciones legales, debates y discusiones generadas alrededor de los servicios de salud del Estado provincial durante los gobiernos peronistas en Córdoba. Dentro de ese conjunto, algunos términos se destacaron por su frecuencia. De las más de treinta menciones en que diferentes actores públicos identificaron a quiénes debían acceder a los bienes oficiales, casi la mitad atribuyó ese beneficio a los enfermos "pobres" y otro quinto mencionó a las personas, la población y las gentes "carentes de recursos". Más del sesenta por ciento de las menciones entonces utilizó los términos "pobre" y "carente de recursos". Frente a los cuales se erigían los "pudientes" y los "ricos".

Para 1947 en los dispensarios provinciales eran exigidos certificados de pobreza para ser atendidos ${ }^{56}$ y para obtener medicamentos sucedía algo similar. Según el senador oficialista Moyano Trebucq, el Ministerio de Salud provincial distribuía estreptomicina entre la "gente pobre" con certificado de pobreza o certificados médicos y que el mismo los había "suscripto muchas veces, y así nos hacemos responsables del destino que se da a la droga."57 Para 1952, leemos en un reglamento de la guardia del Hospital Eva Perón (ex Córdoba), que a menos que se tratara de una urgencia que ameritara internación, todos los enfermos debían pasar por el Servicio Social Médico para que, una vez fichados, fueran dirigidos al servicio correspondiente. ${ }^{58}$

Algo semejante pareció suceder en el diseño y la ejecución de otras políticas sanitarias provinciales y nacionales. De acuerdo al decreto de creación de la Secretaría de Salud Pública de la Nación, el Estado debía asegurar el acceso a las prestaciones médicas de las "clases modestas". Y en el Plan Analítico de Salud Pública el término "pudiente" era utilizado para delimitar a los beneficiarios de la medicina asistencial estatal. Eran los "no pudientes" los poseedores de un derecho social y, por medio de la semisocialización de la medicina, los médicos estarían obligados a atender diariamente a la "población indigente y también de aquellos cuyas rentas a determinarse, no les capaciten para poder hacer frente a los gastos que demanda una enfermedad” (Secretaría de Salud Pública de la Nación, 1947, t. 1:73, 150, 286). De acuerdo al Segundo Plan Quinquenal, "la medicina asistencial que presté el Estado siempre será gratuita y será para la población que no cuente con recursos suficientes" (Presidencia de la Nación, 1952: 83). Esta disposición era replicada en el Plan Provincial cordobés ${ }^{59}$ y en los discursos del poder ejecutivo tucumano. ${ }^{60}$ En un formulario impreso para los ingresantes al hospital nacional de antituberculosos de Santa María, bajo dependencia del Ministerio Nacional de

56 AGPC, Gobierno, 1947, t. 5, f. 1.

57 Diario de Sesiones de la Cámara de Senadores de la Provincia de Córdoba, 1950, p. 525.

58 Decreto 198/d/52. AGPC, Decretos de Salud, 1952, t. 50, f.126r, Servicio de Guardia Hospital Eva Perón. 59 Diario de Sesiones de la Cámara de Senadores de la Provincia de Córdoba, 1952, p. 1215.

60 En un mensaje a la legislatura de Tucumán, el gobernador afirmaba "aseguraré la asistencia médica gratuita a toda la población de escasos recursos..." entre otros objetivos de política sanitaria. (5 de mayo de 1953), La Gaceta, Tucumán, p. 3. 
Salud Pública, se leía en 1950, "Por encontrarme enferm... atacad... de Tuberculosis y careciendo de recursos para mi asistencia médica necesaria...”1

De todos modos, durante el peronismo comenzaron a utilizarse otros adjetivos para asegurar la focalización, así el término "pobre" comenzó a ser sustituido por "carentes de recursos". Este último podía tener una carga valorativa menos estigmatizadora, pero contenía igual capacidad para focalizar los servicios de salud al excluir a los "pudientes". También son visibles otros sustitutos. El relevamiento de publicaciones militantes como Mundo Peronista da cuenta del reemplazo del término "pobre" por el de "humilde", vocablo más habitual en la retórica oficial de esa etapa. Aparecen comentarios en los que se habla de los "humildes", de "las zonas más humildes del país" y de los "sectores humildes". Pero más allá de la sustitución, la operación clasificatoria "pudientes" "ricos"/"pobres" "humildes" siguió permeando los testimonios oficiales.

En ese contexto, una de las novedades de la emergencia del peronismo posiblemente no fue eliminar la carga estigmatizante en esta segmentación de la población, sino permitir a los trabajadores evitar esa operación de clasificación marcada culturalmente por su condición de carencia. A partir del peronismo, los trabajadores pudieron acceder crecientemente a sus propias prestaciones mutualistas, ajenas a los servicios asistenciales del Estado y a los estigmas asociados a la pobreza. Las nuevas condiciones de consumo y bienestar permitieron escindir a buena parte de la población trabajadora de la calificación de "pobre" al gestionar su salud.

La permanencia de este binomio clasificatorio tuvo una larga vida, todavía en 1958, en la Cámara de Diputados de la Nación, ingresó un reglamento de aranceles hospitalarios con despacho positivo de la Comisión de Asistencia Social y Salud Pública, en cuyo segundo artículo aseveraba que se eximiría del mismo a los "pobres de solemnidad", inserción tachada por la de "carentes de recursos" que "justifiquen fehacientemente dicho estado mediante la exhibición de un certificado de pobreza otorgado por autoridad competente..." La carencia de recursos se fijaría de acuerdo al salario y sería variable a lo largo del país, incluyendo a quienes "tienen lo mínimo para vivir con decoro y dignidad, pero a los cuales basta el menor desequilibrio presupuestal para caer en falencia social”. ${ }^{62}$

\section{Conclusiones}

El aumento de la esperanza de vida al nacer, la caída de los índices de mortalidad bruta y mortalidad infantil, la modificación de los contornos urbanos y el acceso efectivo a servicios de salud, educación y alimentación modificaron las condiciones de vida material de la población a partir de la entreguerras. Ahora bien, muchas de las modalidades de distribución de esos servicios, lejos de apuntalar

61 AGPC, 1949, t. 3, s/f, Exp. 194-C-49.

62 Patrimonio Legislativo, Cámara de Diputados de la Nación Argentina, Exp. 433-d-1958. 
nociones de igualdad, pertenencia y derechos, fueron en cambio revestidas con valores negativos asociados a la noción de pobreza. Esto implicó que las noveles iniciativas de asistencia social difundieron clasificaciones y modelos de comportamiento creados a instancias de los patrones de interacción jerárquicos y desiguales previamente existentes en la sociedad argentina, los que si bien no produjeron, sí ayudaron a perpetuar.

En ese contexto, la emergencia del Estado como proveedor de servicios sociales se instituyó en oposición a los prestadores identificados con el modelo benéfico asistencial. Esto supuso que la nueva categoría de beneficiario rompiera con nociones clasificatorias previas como las de "pobre válido" o "inválido", propias de la caridad católica. Sin embargo, la estructuración de los servicios asistenciales generó conflictos distributivos de diversa intensidad que favorecieron aquellas posiciones que exigían la limitación de la clientela gratuita del Estado. En el nivel administrativo, esto devino en la generalización de clasificaciones antinómicas, como la de enfermo "pobre" y "pudiente" definidas a partir de su capacidad para proveerse de atención médica a través del mercado. Pero la asignación del adjetivo de "pobre" o "pudiente" implicó la investidura de valores sociales disímilmente apreciados a lo largo de la sociedad. Es necesario recordar que asistir a un hospital o un dispensario público requería ser investido de la condición de "pobre" o "carente de recursos" y ocupar un lugar dentro de un espacio social atravesado por fronteras simbólicas con importantes consecuencias en la distribución de reconocimiento y valoración social. Algunos funcionarios estatales, médicos y sectores medios habrían estado convencidos de la necesidad de vigilar esa frontera que distanciaba a los "pudientes" de los "pobres" en las modalidades de acceso a los bienes públicos, asegurando tempranamente su focalización. Como consecuencia, en esos años fue instituido un cierre material y simbólico extremadamente eficaz sobre los servicios de salud que en adelante mantuvieron perdurablemente la asociación entre "pobre" y beneficiario del hospital público. 


\section{Bibliografía}

" Adamosvky, E. (2009). Historia de la clase media argentina. Apogeo y decadencia de una ilusión, 1919-2003. Buenos Aires: Planeta.

" Alzate Echeverri, A. M. (2003). Devociones políticas y oratoria salubrista. Sobre un plan de reforma hospitalaria en la Nueva Granada (1790). Historia Crítica, 23, pp. 51-68.

" Alzate Echeverri, A. M. (2008). Militares, marineros y pobres enfermos. Contribución a la historia del hospital San Juan de Dios de Cartagena de Indias (siglo XVIII). Asclepio, LX (1), pp. 203-236.

" Auyero, J. (2013). Pacientes del Estado. Buenos Aires: Eudeba.

" Belmartino, S. et al. (1988). Corporación médica y poder en salud. Argentina, 1920-1945. Centro de Estudios Sanitarios y Sociales: Rosario.

" Bourdieu, P. (1998). La distinción. Criterio y bases sociales del gusto. Madrid: Taurus.

" Bourdieu, P. (1985). ¿Qué significa hablar? Economía de los intercambios lingüísticos. Madrid: Akal.

"Cabred, D. (s/f). Discursos sobre asilos y hospitales regionales en la República Argentina. Buenos Aires: Talleres Gráficos J. Weiss y Preusche.

"Cetrángolo, A. (1935). La crisis de la profesión médica. Revista Médica. Círculo Médico de Córdoba, pp. 541-522.

" Corrigan, P. y Sayer D. (2007 [1985]). El Gran Arco: La formación del Estado inglés como revolución cultural. M. L. Lagos y P. Calla (comp.), Antropología del Estado. Dominación y prácticas contestatarias en América Latina. La Paz: INDH/PNUD, pp. 39-113.

"De la Cadena, M. (2007). Formaciones de indianidad. Articulaciones raciales, mestizaje y nación en América Latina. Envión.

" Fernández, M. E. (2017) (Coord.). La salud: instituciones, espacios y actores. Buenos Aires: Imago Mundi.

" Foucault, M. (2006). Seguridad, territorio, población: Curso en el Collège de France: 19771978. Buenos Aires: FCE.

" Garzón, R. (1934). Memoria del Consultorio Dermatológico. Revista Médica. Circulo Médico de Córdoba, pp. 116-124.

" Goffman, E. (2006). Estigma. La identidad deteriorada. Buenos Aires: Amorrortu editores.

" Golbert, L. (2008). Las políticas sociales antes y después de la Fundación Eva Perón. En C. Barry, K. Ramacciotti y A. Valobra (ed.), La Fundación Eva Perón y las mujeres: entre la provocación y la inclusión. Buenos Aires: Biblos.

" Graves, P. M. (2009). A Blessing or a Curse? Working-Class Attitudes to State Welfare Programmes in Britain 1919-1939. Labour History Review, 74 (2), pp. 160-184.

" Grimson, A. (2017). Raza y clase en los orígenes del peronismo: Argentina, 1945. Desacatos 55, pp. 110-127.

" Herrero, A J. y Prece, A. O. (1935). Dependencia del médico con la situación económica. Revista Médica. Círculo Médico de Córdoba, pp. 28-38.

"Infeld, Ana (2009). Pobres y prostitutas. Políticas sociales, control social y ciudadanía en Comodoro Rivadavia (1929-1944). Rosario: Prohistoria. 
" Karush, M. B. (2013). Cultura de clase. Radio y cine en la creación de una Argentina dividida (1920-1946). Buenos Aires: Ariel.

" Lamont, M. y Virág, M. (2002). The Study of Boundaries in the Social Sciences. Anuual Review of Sociology, 28, pp. 167-195.

" Lorenzo del Río, M. D. (2011). El Estado como benefactor. Los pobres y la asistencia pública en la ciudad de México. México: El Colegio de México/El Colegio Mexiquense.

" Mauss, M. y E. Durkheim (1996). Sobre algunas formas primitivas de clasificación. Contribución al estudio de las representaciones colectivas. E. Durkheim, Clasificaciones primitivas (y otros ensayos de antropología positiva). Madrid: Ariel.

"Moreyra, B. (2009). Cuestión social y políticas sociales en la Argentina. La modernidad periférica. Córdoba, 1900-1930. Bernal: UNQ.

" Ortiz Bergia, M. J. (2015). La temprana descentralización de los servicios de salud en la Argentina: la construcción del sistema sanitario en Córdoba, 1930-1955. História, Ciências, Saúde - Manguinhos, 22 (2), pp. 559-575.

" Ortiz Bergia, M. J. (2009) De caridades y Derechos. La construcción de políticas sociales en el interior argentino. Córdoba (1930-1943). Córdoba: Centro de Estudios Históricos.

" Parolo, M. P. (2005/2006). Nociones de pobreza y políticas hacia los pobres en Tucumán en la primera mitad del siglo XIX. Población \& Sociedad, 12/13, pp. 133-163.

"Presidencia de la Nación, Subsecretaría de Informaciones (1952). Segundo Plan Quinquenal. Buenos Aires.

"Provincia de Córdoba (1940). Memoria del Ministerio de Gobierno correspondiente al ejercicio de 1939. Córdoba.

" Provincia de Santa Fe. Dirección General de Higiene (1933). Memoria del año 1933. Santa Fe.

"Provincia de Santa Fe. Dirección General de Higiene (1938). Memoria del año 1938. Santa Fe.

"Quesada, E. (1917). La vida colonial cordobesa y la práctica de la medicina. Revista de la Universidad, 4 (1), pp. 62-89.

" Ramacciotti, K. (2009). La política sanitaria del peronismo. Buenos Aires: Biblos.

" Rebagliati, L. (2016). Del "pobre afligido" al "vicioso holgazán": concepciones de pobreza en Buenos Aires (1700-1810). Anuario del Instituto de Historia Argentina, 16 (2), (Consultado en línea en https://www.anuarioiha.fahce.unlp.edu.ar/article/view/ IHAe027/7929, 16/3/17).

" República Oriental del Uruguay (1942). Memoria del Ministerio de Salud Pública 19381942. Uruguay.

"Secretaría de Salud Pública de la Nación (|947). Plan analítico de salud pública. Buenos Aires.

" Tilly, C. (2000). La desigualdad persistente. Buenos Aires: Manantial. 